\title{
What to see when you are looking at confusion: a review of the neuroimaging of acute encephalopathy
}

\author{
Raoul Sutter, ${ }^{1,2,3,4}$ Peter W Kaplan ${ }^{1,2}$
}

\begin{abstract}
- Additional material is
published online only. To view please visit the journal online (http://dx.doi.org/10.1136/ jnnp-2014-308216)

1 Division of Neurosciences Critical Care, Department of Anesthesiology and Critical Care Medicine, Johns Hopkins University School of Medicine, Baltimore, Maryland, USA ${ }^{2}$ Department of Neurology, Johns Hopkins Bayview Medical Center, Baltimore, Maryland, USA

${ }^{3}$ Clinic of Intensive Care Medicine, University Hospital Basel, Basel, Switzerland ${ }^{4}$ Department of Neurology, University Hospital Basel, Basel, Switzerland
\end{abstract}

\section{Correspondence to}

Dr Raoul Sutter, Department of Neurology and Intensive Care Units, University Hospital Basel, Petersgraben 4, Basel 4031, Switzerland;

Raoul.Sutter@usb.ch

Institution where the work was performed: Division of Neurosciences Critical Care, Johns Hopkins University School of Medicine.

Received 31 March 2014 Revised 9 July 2014 Accepted 16 July 2014 Published Online First 4 August 2014

\section{ABSTRACT}

Acute encephalopathy is a clinical conundrum in neurocritical care facing physicians with diagnostic and therapeutic challenges. Encephalopathy arises from several concurrent causes, and delayed diagnosis adds to its grim prognosis. Diagnosis is reached by melding clinical, neurophysiological and biochemical features with various neuroimaging studies. We aimed to compile the pathophysiology of acute encephalopathies in adults, and the contribution of cerebral CT, MRI, MR spectroscopy (MRS), positron emission tomography (PET) and single-photon emission CT (SPECT) to early diagnosis, treatment and prognostication. Reports from 1990 to 2013 were identified. Therefore, reference lists were searched to identify additional publications. Encephalopathy syndromes best studied by neuroimaging emerge from hypoxic-ischaemic injury, sepsis, metabolic derangements, autoimmune diseases, infections and rapidly evolving dementias. Typical and pathognomonic neuroimaging patterns are presented. Cerebral imaging constitutes an important component of diagnosis, management and prognosis of acute encephalopathy. Its respective contribution is dominated by rapid exclusion of acute cerebral lesions and further varies greatly depending on the underlying aetiology and the range of possible differential diagnoses. CT has been well studied, but is largely insensitive, while MRI appears to be the most helpful in the evaluation of encephalopathies. MRS may provide supplementary biochemical information and determines spectral changes in the affected brain tissue. The less frequently used PET and SPECT may delineate areas of high or low metabolic activity or cerebral blood flow. However, publications of MRS, PET and SPECT are limited only providing anecdotal evidence of their usefulness and sensitivity.

\section{INTRODUCTION}

Encephalopathy is a major cause of morbidity and mortality, particularly in the intensive care unit and in postsurgical patients. Acute encephalopathy appears in many forms, producing a constellation of abnormalities with myriad clinical features, dysfunctions reflected in tests on body fluids, abnormalities on EEG and a number of imaging abnormalities. Although individual or even aggregates of tests may not yield a specific diagnosis, they summate to produce a picture that can help the clinician in a directed management plan. Over the past few decades we have witnessed major advances in promising neuroimaging modalities and emerging new acquisition techniques with an increasing availability. Among neuroimaging techniques, including cerebral CT, brain MRI, single-photon emission CT (SPECT) and positron emission tomography (PET), several have yielded identifiable abnormalities leading to the causes of acute brain dysfunction hence expediting appropriate treatment. In light of the increasing and diverse imaging tools, guidance regarding their diagnostic and prognostic yield in the clinically challenging setting of acute encephalopathy as well as their impact on patient management is evermore important for the clinician.

We therefore aimed to compile the current evidence and to provide a compendium of typical, and in some cases pathognomonic, imaging patterns for the clinician. We review the essential pathophysiology of selected, well-recognised acute encephalopathies in adults, and their respective neuroimaging correlates. Further, we aimed to compile the prognostic value of neuroimaging in these settings.

\section{DEFINITION OF ENCEPHALOPATHY}

There is no international consensus regarding the distinction between encephalopathy, delirium and confusional states. Terms such as 'delirium', 'acute brain syndrome', 'acute brain dysfunction', 'acute confusional state', and 'toxic or metabolic encephalopathy' are often used synonymously and in an inconsistent manner. The term 'encephalopathy' encompasses a wide variety of reversible or permanent, diffuse or multifocal brain dysfunctions caused by myriad physiological derangements ranging from acute structural brain alteration (eg, trauma, ischaemia, haemorrhage, tumour, etc) to non-structural metabolic, toxic and/or infectionrelated cerebral dysfunction. However, acute encephalopathy, is defined by the American Psychiatric Association as altered consciousness with change in cognition and/or with a perceptual disturbance developing over hours or days and that which was not better accounted for by a preexisting or evolving chronic dementia. ${ }^{1}$

\section{IMAGING TECHNIQUES}

CT uses computer-processed X-rays to produce tomographic images of the scanned objects. Digital geometry processing generates three-dimensional images based on a large series of two-dimensional radiographies taken around an axis of rotation.

In diffusion-weighted imaging (DWI), a specific MRI sequence, the intensity of each three-dimensional
To cite: Sutter R,
Kaplan PW. J Neurol

Neurosurg Psychiatry

2015:86:446-459 
image-element (voxel) reflects the estimate of the water diffusion rate in that area. The random motion of water depends on thermal agitation and on its cellular environment. The motion of cellular and intercellular water is hindered primarily by cell membrane boundaries. The diffusion restriction correlates with cellularity of a specific tissue. DWI detects changes of water diffusion that may indicate (early) pathological alterations of membranes and membrane-bound water transporting proteins. The impedance of water molecules diffusion is quantified using the apparent diffusion coefficient (ADC) value. ADC values can be displayed as a parametric map reflecting the degree of water diffusion through different tissues. MR spectroscopy (MRS) allows studies of metabolic changes in brain tissue by using signals from hydrogen protons to determine the relative concentrations of target brain metabolites.

PET is a functional imaging technique using nuclear medicine producing three-dimensional images of physiological and pathological processes in the body. PET detects $\gamma$-ray pairs emitted indirectly by administered positron-emitting radionuclides, which are linked to a biologically active molecule. Three-dimensional images based on the detected regional concentration of these radionuclide-linked molecules are constructed automatically.

SPECT is a nuclear medicine tomographic imaging technique using $\gamma$-rays. A $\gamma$-emitting radionuclide is administered intravenously and emitted $\gamma$-rays are detected by $\gamma$-cameras. Three-dimensional images are then constructed automatically.

\section{SEARCH STRATEGY AND SELECTION CRITERIA}

We included acute encephalopathies in adolescent and adult patients from metabolic derangements, hypoxic-ischaemic insults, sepsis, autoimmune diseases and rapidly evolving dementias. More chronic or subacute encephalopathies, such as those resulting from multifocal vascular lesions or inborn errors of metabolism were excluded. Further encephalopathies evolving from a large number of exogenous toxins, specific infections other than from herpes simplex virus (HSV), status epilepticus and trauma were excluded, as most of these disorders can indicate a large variety of neuroradiological abnormalities. Studies of functional MRI, diffusion tensor imaging and perfusionweighted MRI were not included, as in most types of acute encephalopathies studies are lacking.

The Medline search engine was used to identify reports published in any language between 1990 and 2013. The search terms included the names of the different encephalopathic syndromes, or pathological conditions used in the headings throughout the manuscript in combination with "computed tomography", "magnetic resonance imaging", "magnetic resonance spectroscopy", "positron emission tomography", "singlephoton emission computed tomography", "CT", "MRI", "MRS", "PET", and "SPECT". In addition, the reference lists of selected reports were searched to identify additional publications.

Publications are summarised and categorised in the online supplementary tables S1-S3.

\section{NEUROIMAGING IN ENCEPHALOPATHIC SYNDROMES \\ Hypoxic-ischaemic encephalopathy}

Pathophysiology: Hypoxic-ischaemic encephalopathy (HIE) usually results from acute circulatory and/or respiratory breakdown and typically affects the cortical and subcortical grey matter structures first. HIE is mainly caused by cardiac arrest, poisoning with carbon monoxide, asphyxiation or drowning. Failure of cerebral oxygenation inhibiting the $\mathrm{Na}^{+}-\mathrm{K}^{+}$pumps leads to the loss of cellular integrity and release of excitotoxic glutamate, followed by overstimulation of $\mathrm{N}$-methyl-D-aspartate (NMDA) receptors, cellular calcium loss mediated via second messengers and damage of the mitochondrial respiratory chain. ${ }^{2}$ Brain reperfusion may cause secondary damage. The severity of injury depends on the duration and degree of circulatory and/or respiratory breakdown, body temperature and serum glucose levels.

Neuroimaging (see online supplementary table S1, figure 1A): Cerebral CT is often normal early on, or may reveal mild loss of the grey-white junction in the first hours after cardiorespiratory arrest (CRA). Conversely, brain MRI may show DWI changes within an hour after CRA. ${ }^{3}$ In the initial $24 \mathrm{~h}$, there may be symmetric decreases in the thalamus and basal ganglia, ${ }^{4}$ loss of differentiation between the grey and white matter, and increases in signal on T2-weighted images. ${ }^{5}$ In the late subacute phase of HIE, there are changes in the white and deep grey matter. ${ }^{6}$

Quantitative whole-brain diffusion-weighted MRI on days 24 after CRA may provide more accurate prognosis than the neurological examination. ${ }^{7}$ In a study of 10 patients, marked DWI, ADC or fluid-attenuated inversion recovery (FLAIR) changes portended poor outcome in $100 \%{ }^{8}$ Although early cerebral CT is less sensitive than brain MRI, ${ }^{9}$ one study of 53 patients revealed that signs of global cerebral oedema had a false-positive rate of $0 \%$ for death. ${ }^{10}$ In a study of post-CRA patients treated with hypothermia, quantitative whole-brain diffusion-weighted MRI in this time window improved the sensitivity for predicting poor outcome by $38 \%$ while maintaining $100 \%$ specificity as compared with the $72 \mathrm{~h}$ neurological examination $(p=0.021){ }^{7}$ This study was followed by another analysis revealing that DWI $48 \mathrm{~h}$ post-CRA with global ischaemia or focal ischaemia with a total lesion volume of $>20 \mathrm{~mL}$ had a false-positive rate of $0 \%$ for a poor outcome (defined as a cerebral performance category of $3-5)^{11}$ while another study showed a false-positive rate of $23 \%$ of cortical and/or deep grey nuclei lesions on MRI for a Cerebral Performance Category (CPC) of $3-5 .{ }^{12}$ The predictive value of MRI findings is given in table 1. In a small study, MRS distinguished between good and poor outcome with a $100 \%$ specificity. The distinction was made with $90 \%$ sensitivity after $48 \mathrm{~h}$ and became $100 \%$ by days 3 and $4 .{ }^{13}$ Data from brain PET and SPECT studies are of no additional help. Frequent neuroimaging patterns are summarised in table 2.

\section{Sepsis-related encephalopathy}

Pathophysiology: Although the central nervous system (CNS) may be an 'immunologically privileged' organ, separated by the blood-brain barrier from most components of the humoral and cellular immune system, the brain may be exposed to excessive systemic inflammatory responses during sepsis. Cerebral dysfunction reflects the systemic metabolic, inflammatory and haemodynamic disturbances associated with systemic inflammatory response. Septic shock can also cause multifocal necrotising leukoencephalopathy from systemic inflammatory-mediated responses, along with ischaemic brain lesions, haemorrhages and microabscesses. ${ }^{14}$

Neuroimaging (figure 1B): In many patients, cerebral CT and MRI are normal, however, in some studies signs of vasogenic oedema can be detected when autoregulation is disturbed. ${ }^{15}$ With marked sepsis, there may be multiple lesions of cerebral white matter, and ischaemic strokes located in the centrum semiovale. ${ }^{16}$ Signs of ventriculitis with paraventricular ependymal hyperintensities can be present (figure 1B) and periventricular contrast enhancement is often demonstrated best on T1. The patchy white matter lesions are dynamic and change over time. 


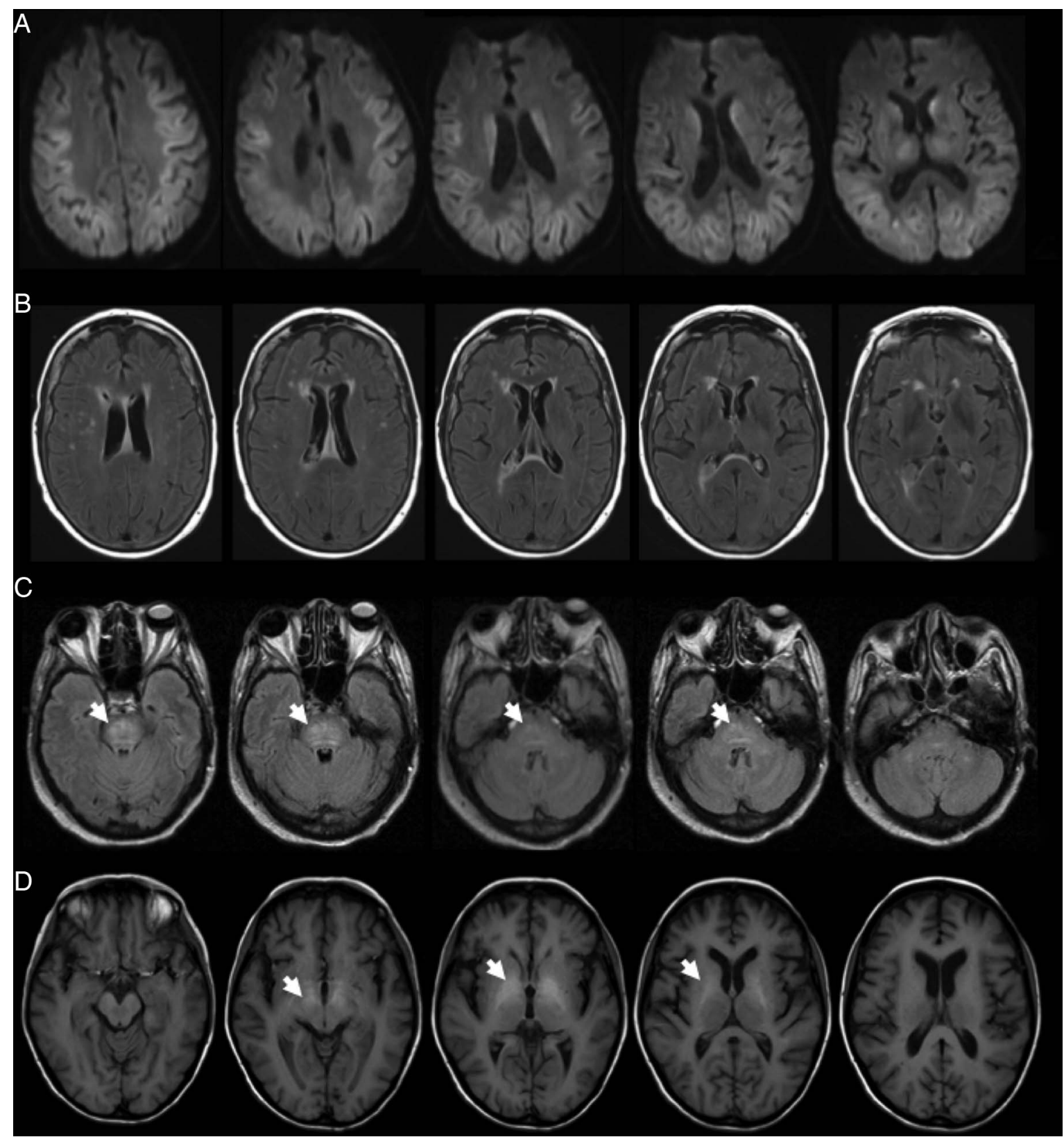

Figure $1 \mathrm{MRI}$ of specific acute encephalopathy syndromes. Serial axial fluid-attenuated inversion recovery sequences (on 1.5-T MRI, except A and D which are diffusion-weighted imaging; on 1.5-T MRI). (A) Hypoxic-ischaemic encephalopathy with hyperintense thalami and cortical grey matter; (B) sepsis-related encephalopathy with bilateral patchy hyperintensities in the deep white matter and ependymal hyperintensities along the ventricules (ie, ventriculitis); (C) central pontine myelinolysis (central pontine hyperintensities in the pons (arrows)); and (D) acute hepatic encephalopathy with hyperintensities from a cytotoxic oedema in the globus pallidus (arrows).

There are no sufficient data on MRS, SPECT and PET in humans. Frequent neuroimaging patterns are summarised in table 2 .

\section{Uraemic encephalopathy}

Pathophysiology: The exact pathophysiology of uraemic encephalopathy is unknown, but reversible ischaemic changes, disorders of cerebral metabolism, and the direct effect of uraemic toxins on intracerebral vascular autoregulation have been implicated. ${ }^{17}$

Neuroimaging (see online supplementary table S2): Cerebral CT findings are usually normal, although some reveal attenuation changes in bilateral basal ganglia and internal capsules. ${ }^{18}$ Brain MRI has shown T1 hypointensities and T2, FLAIR and
DWI hyperintensities in the same areas, along with changes in cortical zones in some cases. ${ }^{17}$ Individual reports in patients without diabetes with uraemia showed extensive supratentorial white matter changes sparing basal ganglia and cortical involvement $^{19}$ with diffusion restrictions in some patients. Following haemodialysis, many of these imaging and clinical abnormalities may regress. Nonetheless, in patients with relapsing and remitting uraemia, encephalopathy and concurrent cerebral atrophy progress and the basal ganglia may also be affected resulting in disturbed extrapyramidal movements. ${ }^{20}$ MRS reveals a decreased $\mathrm{N}$-acetyl-aspartate peak and an elevated lactate peak in the basal ganglia when extrapyramidal movement abnormalities occur. ${ }^{21}$ Brain F-18 fluorodeoxyglucose (FDG)-PET in patients with extrapyramidal movement disorders may uncover 
Table 1 Most consistent and pathognomonic neuroimaging patterns in the literature and their predictive value (data from the online supplementary tables S1-S3)

Hypoxic-ischaemic encephalopathy

CT Global cerebral oedema

- Decrease of putaminal, cortical and corticomedullary contrast

MRI

- Diffuse signal abnormalities in the cortex and subcortical areas or effacement of the sulci

- Lower whole-brain and regional median ADC

- DWI and FLAIR cortical multilobar, or diffuse lesion pattern

- $A D C<650 \times 10^{6} \mathrm{~mm}^{2} / \mathrm{s}$

- DWI with global ischaemia or focal ischaemia with lesion volume $>20 \mathrm{~mL}$

- T2 and DWI changes in the cerebral cortex and the deep grey matter

PET Hypometabolism frontal, parietal including the precuneus, in the posterior cingulate gyrus, and in the occipital areas. Hypermetabolism in the insulas, cerebellum and brainstem

Sepsis-related encephalopathy

MRI Vasogenic oedema can be detected when autoregulation is disturbed

- With marked sepsis, there may be multiple lesions of cerebral white matter, and ischaemic strokes located in the centrum semiovale. The patchy white matter lesions are dynamic and change over time

- Periventricular contrast enhancement may often be demonstrate best on T1

- Signs of ventriculitis with paraventricular ependymal hyperintensities can be present

Uraemic encephalopathy

CT Hypodense basal ganglia and capsules

MRI T2, FLAIR and DWI hyperintense basal ganglia, capsules and inconsistently in cortical areas

- Additional involvement of white matter and cerebral peduncles, occipital lobes and thalami

- Decrease of $\mathrm{N}$-acetyl-aspartate and the presence of lactate on MRS

PET Decreased glucose metabolism in basal ganglia

Hyperammonaemic encephalopathy

MRI T2, FLAIR and DWI cortical hyperintensities of the insula and cingulum

- Increased glutamine and glutamate; low myoinositol and choline on MRS

Hepatic encephalopathy

CT Usually normal

MRI T1 hyperintensities in the globus pallidus and, less frequent, in the substantia nigra and the midbrain tegmentum

- FLAIR and DWI hyperintense thalami, posterior limbs of the internal capsule, periventricular region, dorsal brain stem and diffuse cortical involvement in 1 study of 20 patients

- Connectivity: decreased in the caudate of the anterior/middle cingulate gyrus; increased in the caudate of the left motor cortex; reduced between the putamen and the anterior cingulate gyrus, right insular lobe, inferior frontal gyrus, left parahippocampal gyrus and the anterior lobe of the right cerebellum; increased between the putamen and right middle temporal gyrus

- Increased glutamate/glutamine ratio and low myoinositol and choline on MRS

- Diminished choline and elevated glutamate/glutamine ratio in the parieto-occipital cortex on MRS

SPECT High blood flow in the cerebellum, basal ganglia and cerebral cortex

- Alteration of striatal D2-receptor binding and dopamine reuptake

PET Hypoperfusion of the superior and middle frontal gyri, and inferior parietal lobules

- Increased peripheral benzodiazepine binding sites prefrontal and striatal in patients with cirrhosis

Wernicke's encephalopathy

CT Hypodense paraventricular thalamic regions with or without contrast enhancement and, No clear evidence less frequent, hypodense periaqueductal regions, tectum of the midbrain and tegmentum of the pons

MRI $\quad \mathrm{T} 2$ and FLAIR hyperintense periaqueductal and medial thalamic regions. Less-frequent hyperintense mammillary bodies, periaqueductal regions, hypothalamus, tectum and cerebellum

- Minimised mammillary bodies

- Contrast-enhanced mammillary bodies is related to alcohol abuse

- Atrophic mammillary bodies and cerebellar vermis (chronic phase)

- Thalamic lactate increase and low $\mathrm{N}$-acetyl-aspartate/creatine on MRS

SPECT Hypoperfusion frontoparietal and in the right basal ganglia
No clear evidence

No clear evidence

No clear evidence

No clear evidence
Global oedema false-positive rate $0 \%$. Higher putaminal, cortical and corticomedullary contrast was associated with Cerebral Performance Category (CPC) 4-5

All patients with these findings died

Patients with modified Rankin Scale (mRS) $>3$ have lower whole-brain and regional $A D C$

False-positive rate $0 \%$ for death, profound cognitive impairment persistent vegetative state or severe physical impairment

No clear evidence

No clear evidence

No clear evidence

No clear evidence

No clear evidence

No clear evidence

No clear evidence

Correlations between the corticostriatal connectivity and neuropsychological performances, but not between the striatal connectivity and globus pallidus signal intensity 
Table 1 Continued

\begin{tabular}{|c|c|c|}
\hline Imaging & Patterns & Predictive value for poor outcome \\
\hline \multicolumn{3}{|c|}{ Hypoglycaemic encephalopathy } \\
\hline $\mathrm{CT}$ & $\begin{array}{l}\text { Enhancing, hypodense basal ganglia, cerebral cortex, hippocampus and substantia } \\
\text { nigra }\end{array}$ & No clear evidence \\
\hline MRI & $\begin{array}{l}\text { T2 and FLAIR hyperintensities in the caudate, lenticular nuclei, cerebral cortex, } \\
\text { substantia nigra, hippocampus and internal capsules } \\
\text { In few patients DWI hyperintense white and deep grey matter and splenium of the } \\
\text { corpus callosum }\end{array}$ & $\begin{array}{l}\text { Associated with vegetative state or severe disability } \\
\text { in two case series }\end{array}$ \\
\hline \multicolumn{3}{|c|}{ Hyperglycaemic encephalopathy } \\
\hline $\mathrm{CT}$ & Hyperdense putamen and/or caudate nucleus & No clear evidence \\
\hline MRI & Unilateral or bilateral T1 hyperintensities in the striatum (mostly putamen) & No clear evidence \\
\hline SPECT & Hypoperfusion of the basal ganglia & No clear evidence \\
\hline \multicolumn{3}{|c|}{ Hyponatraemic/hypernatraemic encephalopathies (pontine or extrapontine myelinolysis) } \\
\hline $\mathrm{CT}$ & - Normal in a few patients and hypodense pontine lesions in the others & No clear evidence \\
\hline MRI & $\begin{array}{l}\text { T2, FLAIR and DWI hyperintense lesions of the pons. Less frequent lesions of the } \\
\text { thalamus, midbrain, cortical grey matter, hippocampus, caudate, putamen and } \\
\text { middle cerebral peduncle } \\
\text { T2 hyperintensities may be distributed along the crowns and sides of the cerebral } \\
\text { gyri }\end{array}$ & No clear evidence \\
\hline SPECT & $\begin{array}{l}\text { Decreased striatal dopamine transporter binding and pontine hyperperfusion during } \\
\text { recovery }\end{array}$ & No clear evidence \\
\hline
\end{tabular}

a decreased glucose metabolism in the basal ganglia. ${ }^{22}$ Frequent neuroimaging patterns are summarised in table 3 .

\section{Hyperammonaemia-related encephalopathy}

Pathophysiology: Hyperammonaemic encephalopathy (HAE) results from the purported toxic effect of ammonia (or other

Table 2 Neuroimaging patterns in hypoxic-ischaemic and sepsis-related encephalopathy

\begin{tabular}{|c|c|}
\hline Imaging & Patterns \\
\hline \multicolumn{2}{|c|}{ Hypoxic-ischaemic encephalopathy } \\
\hline $\mathrm{CT}$ & $\begin{array}{l}\text { - Global cerebral oedema } \\
\text { - Decrease of putaminal, cortical and corticomedullary contrast }\end{array}$ \\
\hline MRI & $\begin{array}{l}\text { Diffuse signal abnormalities in the cortex and subcortical areas or } \\
\text { effacement of the sulci } \\
\text { Lower whole-brain and regional median ADC } \\
\text { DWI and FLAIR cortical multilobar, or diffuse lesion pattern } \\
\text { ADC }<650 \times 10^{6} \mathrm{~mm}^{2} / \mathrm{s} \\
\text { DWI with global ischaemia or focal ischaemia with lesion volume } \\
>20 \mathrm{~mL} \\
\text { T2 and DWI changes in the cerebral cortex and the deep grey } \\
\text { matter }\end{array}$ \\
\hline PET & $\begin{array}{l}\text { Hypometabolism frontal, parietal including the precuneus, in the } \\
\text { posterior cingulate gyrus, and in the occipital areas. Hypermetabolism } \\
\text { in the insulas, cerebellum and brainstem }\end{array}$ \\
\hline \multicolumn{2}{|c|}{ Sepsis-related encephalopathy } \\
\hline MRI & $\begin{array}{l}\text { Vasogenic oedema can be detected when autoregulation is } \\
\text { disturbed } \\
\text { With marked sepsis, there may be multiple lesions of cerebral } \\
\text { white matter, and ischaemic strokes located in the centrum } \\
\text { semiovale. The patchy white matter lesions are dynamic and } \\
\text { change over time } \\
\text { Periventricular contrast enhancement may often be demonstrated } \\
\text { best on T1 } \\
\text { Signs of ventriculitis with paraventricular ependymal } \\
\text { hyperintensities can be present }\end{array}$ \\
\hline
\end{tabular}

$A D C$, apparent diffusion coefficient; DWI, diffusion-weighted imaging; FLAIR,

fluid-attenuated inversion recovery; PET, positron emission tomography. compounds that co-occur with ammonia), a product of the amino acid metabolism. ${ }^{23}$ Ammonia may accumulate when its degradation in the liver is impaired mainly as a result of liver diseases, or by its overproduction in pathological conditions including multiple myeloma, urease-producing bacteria, or drugs (eg, divalproate sodium) that inhibit metabolic pathways. $^{23}$ Increased glutamine production during ammonium clearance, changes in osmolarity and death of astrocytes with brain oedema may be the causes of encephalopathy. ${ }^{23}$

Neuroimaging (see online supplementary table S2): Cerebral CT may not be particularly helpful. In contrast, two case series of brain MRI findings have revealed bilateral changes in the T2-weighted images and FLAIR sequences of the insula, cingulum, and more diffuse cortical regions using diffusion-weighted sequences. $^{24}$ MRS can show increases in glutamate and

Table 3 Neuroimaging patterns in uraemic and hyperammonaemic encephalopathy

\begin{tabular}{|c|c|}
\hline Imaging & Patterns \\
\hline \multicolumn{2}{|c|}{ Uraemic encephalopathy } \\
\hline СТ & Hypodense basal ganglia and capsules \\
\hline MRI & $\begin{array}{l}\text { T2, FLAIR and DWI hyperintense basal ganglia, capsules and } \\
\text { inconsistently in cortical areas } \\
\text { - Additional involvement of white matter and cerebral peduncles, } \\
\text { occipital lobes and thalami } \\
\text { Decreased } N \text {-acetyl-aspartate and an elevated lactate in the basal } \\
\text { ganglia on MRS }\end{array}$ \\
\hline PET & Decreased glucose metabolism in basal ganglia \\
\hline \multicolumn{2}{|c|}{ Hyperammonaemic encephalopathy } \\
\hline СТ & Usually normal \\
\hline MRI & $\begin{array}{l}\text { T2, FLAIR and DWI cortical hyperintensities of the insula and } \\
\text { cingulum } \\
\text { Increased glutamine and glutamate; low myoinositol and choline } \\
\text { on MRS }\end{array}$ \\
\hline
\end{tabular}

DWI, diffusion-weighted imaging; FLAIR, fluid-attenuated inversion recovery; MRS, MR spectroscopy; PET, positron emission tomography. 
glutamine, decreases in myoinositol and choline possibly representing compensatory mechanisms. ${ }^{25}$ In HAE with cirrhosis, PET demonstrates decreased cerebral blood flow with a fall in cerebral metabolic rate for oxygen by $20-40 \%$ compared with normal controls. ${ }^{26}$ Studies on SPECT in HAE are lacking. Frequent neuroimaging patterns are summarised in table 3.

\section{Hepatic encephalopathy}

Pathophysiology: Hepatic encephalopathy (HE) emerges from decompensated cirrhosis or acute hepatic failure. Astrocytic swelling resulting from an accumulation of metabolites is considered a major contributor ${ }^{25}$ (see Hyperammonaemia-related encephalopathy section, above).

Neuroimaging (see online supplementary table S3, figure 1D): There are few neuroimaging studies on acute HE. ${ }^{25}$ Cerebral CT may be normal, or shows non-specific changes in the tectal regions, anterior pituitary and subthalamic nucleus. On MRI, there may be T2 prolongation in the periventricular white matter, thalami, internal capsules, corticospinal tracts and cerebral cortex. ${ }^{2527}$ In acute fulminant hepatic failure, the reversible oedema is astrocytic and mostly affects the globus pallidus, even though the DWI can resemble the (usually fatal) appearance in HIE. Changes in the glutamate/glutamine ratio with decreased myoinositol and choline are seen with $\mathrm{MRS}^{25}$ and can regress following liver transplantation. There is no evidence for the added diagnostic or prognostic value of MRS, PET and SPECT in HE. Cerebral SPECT may detect an increase in cerebellar, cortical and basal ganglia blood flow, ${ }^{28}$ and changes in dopamine D2-receptor binding and reuptake. ${ }^{29}$ Some patients with cirrhosis have decreased perfusion in the inferior parietal lobules, and the middle and superior frontal gyri. ${ }^{30}$ Many of these changes regress following treatment.

\begin{tabular}{ll} 
Table 4 Neuroimaging patterns in hepatic encephalopathy \\
\hline Imaging Patterns \\
\hline Hepatic encephalopathy \\
CT Usually normal \\
MRI \\
T1 hyperintensities in the globus pallidus and, less frequent, in the \\
substantia nigra and the midbrain tegmentum \\
FLAIR and DWI hyperintense thalami, posterior limbs of the \\
internal capsule, periventricular region, dorsal brain stem and \\
diffuse cortical involvement in 1 study of 20 patients \\
In acute hepatic encephalopathy the widespread grey matter \\
changes on FLAIR and DWI are often reversible, in contrast to \\
anoxic-ischaemic encephalopathy \\
Connectivity: decreased in the caudate of the anterior/middle \\
cingulate gyrus; increased in the caudate of the left motor cortex; \\
reduced between the putamen and the anterior cingulate gyrus, \\
right insular lobe, inferior frontal gyrus, left parahippocampal \\
gyrus and the anterior lobe of the right cerebellum; increased \\
between the putamen and right middle temporal gyrus \\
Increased glutamate/glutamine ratio and low myoinositol and \\
choline on MRS \\
Diminished choline and elevated glutamate/glutamine ratio in the \\
parieto-occipital cortex on MRS \\
- High blood flow in the cerebellum, basal ganglia and cerebral \\
cortex \\
Alteration of striatal D2-receptor binding and dopamine reuptake \\
P \\
Hypoperfusion of the superior and middle frontal gyri, and inferior \\
parietal lobules \\
Increased peripheral benzodiazepine binding sites prefrontal and \\
striatal in patients with cirrhosis
\end{tabular}

DWI, diffusion-weighted imaging; FLAIR, fluid-attenuated inversion recovery; MRS, MR spectroscopy; PET, positron emission tomography; SPECT, single-photon emission CT.
In a recent study, MRI lesion severity correlated moderately with the clinical outcome but was not significant after Bonferroni correction for multiple comparisons. ${ }^{27}$ The predictive value of MRI findings is given in table 1. Frequent neuroimaging patterns are summarised in table 4 .

\section{Hypoglycaemic and hyperglycaemic encephalopathy \\ Hypoglycaemic encephalopathy}

Pathophysiology: An acute decrease in serum glucose levels usually arises from an excess of hypoglycaemia inducing drugs, exogenous or endogenous insulin. This may release aspartate, an excitatory neurontransmitter, after a decline of the cell membrane ATPase pump activity.

Neuroimaging (see online supplementary table S2): Cerebral CT can show enhancing hypodensities in the basal ganglia, cerebral cortex, hippocampus and substantia nigra. ${ }^{31}$ Brain MRI is usually more revealing and demonstrates hyperintense T2 signals and diffusion restrictions are believed to represent neuronal death and cytotoxic oedema in the posterior limb of the internal capsules, hippocampi, the basal ganglia and cortical areas. ${ }^{32}$ The splenium of the corpus callosum may also show signal changes. ${ }^{32}$ Data regarding MRS are lacking and there are only few studies using SPECT, or PET with inconclusive findings.

Nonetheless, case series suggest poor outcome with diffuse and extensive DWI changes in the basal ganglia and deep white matter. ${ }^{32} 33$ In two case series of patients with bilateral symmetrical grey and/or white matter lesions, $100 \%$ remained severely disabled or in a vegetative state. ${ }^{32} 33$ The predictive value of MRI findings is given in table 1 . Frequent neuroimaging patterns are summarised in table 5 .

Table 5 Neuroimaging patterns in hypoglycaemic and hyperglycaemic and hyponatraemic and hypernatraemic encephalopathy

\begin{tabular}{|c|c|}
\hline Imaging & Patterns \\
\hline \multicolumn{2}{|c|}{ Hypoglycaemic encephalopathy } \\
\hline $\mathrm{CT}$ & $\begin{array}{l}\text { Enhancing, hypodense basal ganglia, cerebral cortex, hippocampus } \\
\text { and substantia nigra }\end{array}$ \\
\hline MRI & $\begin{array}{l}\text { T2 and FLAIR hyperintensities in the caudate, lenticular nuclei, } \\
\text { cerebral cortex, substantia nigra, hippocampus and internal } \\
\text { capsules } \\
\text { In few patients DWI hyperintense white and deep grey matter and } \\
\text { splenium of the corpus callosum }\end{array}$ \\
\hline \multicolumn{2}{|c|}{ Hyperglycaemic encephalopathy } \\
\hline $\mathrm{CT}$ & Hyperdense putamen and/or caudate nucleus \\
\hline MRI & $\begin{array}{l}\text { Unilateral or bilateral } \mathrm{T} 1 \text { hyperintensities in the striatum (mostly } \\
\text { putamen) }\end{array}$ \\
\hline SPECT & Hypoperfusion of the basal ganglia \\
\hline \multicolumn{2}{|c|}{$\begin{array}{l}\text { Hyponatraemic/hypernatraemic encephalopathies (pontine or extrapontine } \\
\text { myelinolysis) }\end{array}$} \\
\hline $\mathrm{CT}$ & $\begin{array}{l}\text { Normal in a few patients and hypodense pontine lesions in the } \\
\text { others }\end{array}$ \\
\hline MRI & $\begin{array}{l}\text { T2, FLAIR and DWI hyperintense lesions of the pons. Less frequent } \\
\text { lesions of the thalamus, midbrain, cortical grey matter, } \\
\text { hippocampus, caudate, putamen and middle cerebral peduncle } \\
\text { T2 hyperintensities may be distributed along the crowns and sides } \\
\text { of the cerebral gyri }\end{array}$ \\
\hline SPECT & $\begin{array}{l}\text { Decreased striatal dopamine transporter binding and pontine } \\
\text { hyperperfusion during recovery }\end{array}$ \\
\hline
\end{tabular}


Figure 2 MRI in Wernicke's encephalopathy. (A) Sagittal diffusion-weighted imaging (on 1.5-T MRI) with typical small corpora mammillaria (corpus albicantia) in Wernicke's encephalopathy (arrow); and (B) axial fluid-attenuated inversion recovery sequences (on 1.5-T MRI) with abnormal hyperintensity of the periaqueductal grey matter (arrows).

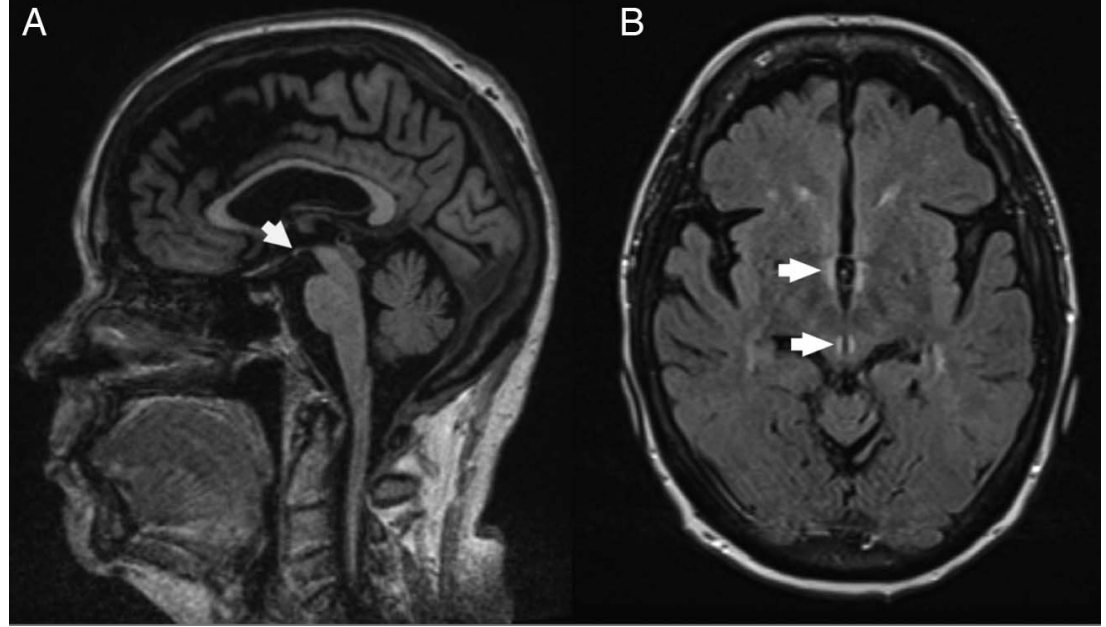

Hyperglycaemic encephalopathy

Pathophysiology: Hyperglycaemia frequently occurs in uncontrolled diabetes mellitus. Diffuse brain dysfunction reflects osmotic derangements affecting the subthalamic area, the putamen and the caudate nucleus. ${ }^{34}$

Neuroimaging (see online supplementary table S2): In 10 patients, cerebral CT showed hyperdense changes in the putamen, while brain MRI revealed T1-weighted hyperintensities with no T2 signal changes in the basal ganglia. ${ }^{34}$ However, these findings are considered inconsistent and in acute hyperosmolar states, neuroimaging (especially MRI) can vary, in the presence of seizures or status epilepticus. Data regarding MRS are lacking. Studies report that brain SPECT shows hypoperfusion in the basal ganglia. ${ }^{34}$ When glucose is corrected, imaging

Table 6 Neuroimaging patterns in Wernicke's and posterior reversible encephalopathy

\begin{tabular}{ll}
\hline Imaging & Patterns \\
\hline Wernicke's encephalopathy \\
CT & Hypodense paraventricular thalamic regions with or without contrast \\
& enhancement and, less frequent, hypodense periaqueductal regions, \\
tectum of the midbrain and tegmentum of the pons & \\
MRI & T2 and FLAIR hyperintense periaqueductal and medial thalamic \\
& regions. Less frequent hyperintense mammillary bodies, \\
& periaqueductal regions, hypothalamus, tectum and cerebellum \\
& Minimised mammillary bodies \\
& Contrast-enhanced mammillary bodies is related to alcohol abuse \\
& Atrophic mammillary bodies and cerebellar vermis (chronic phase) \\
& Thalamic lactate increase and low $N$-acetyl-aspartate/creatine on \\
& MRS
\end{tabular}

SPECT Hypoperfusion frontoparietal and in the right basal ganglia

Posterior reversible encephalopathy

CT Hypodensities in the parieto-occipital subcortical white matter and cerebellum with increased cerebral blood volume, blood flow, and reduced time to peak mainly in the posterior vascular distribution

MRI T2, FLAIR, and DWI hyperintensities in the posterior circulation areas and, less frequent, in the anterior circulation structures. ADC values in areas of abnormal $\mathrm{T} 2$ signal are high

- Contrast enhancement, restrictions on DWI and ADC

- Decrease in $\mathrm{N}$-acetyl-aspartate in patients with normal MRI or reversible MRI changes and only minimal elevation of choline on MRS

ADC, apparent diffusion coefficient; DWI, diffusion-weighted imaging; FLAIR,

fluid-attenuated inversion recovery; MRS, MR spectroscopy; SPECT, single-photon emission CT. abnormalities regress. ${ }^{34}$ SPECT may reveal differences between non-ketotic and ketotic hyperglycaemic encephalopathies. In the former there are often multifocal seizures and extrapyramidal movements that might show corresponding areas of altered metabolism. Frequent neuroimaging patterns are summarised in table 5 .

\section{Hyponatraemic and hypernatraemic encephalopathy} (pontine or extrapontine myelinolysis)

Pathophysiology: The decreased serum osmolality, primarily from acute hyponatraemia promotes a water shift across the blood-brain barrier into the brain tissue resulting in cerebral oedema and severe neurological dysfunction. The increase of interstitial pressure causes an extracellular fluid shift from the brain tissue into the cerebrospinal fluid. The glial cells and neurons lose solutes, promoting an osmotic movement of water from the intracellular to the extracellular space, ${ }^{35}$ subsequent disruption of the blood-brain barrier, and loss of oligodendroglia in the basis pontis. The rapid correction/change of serum sodium levels causes central pontine myelinolysis (CPM) - an effect, which is not entirely understood. This osmotic demyelination can also evolve more diffusely to involve extrapontine regions of the CNS (ie, extrapontine myelinolysis).

Neuroimaging (see online supplementary table S2, figure 1C): Cerebral CT reveals evidence of demyelination in $\sim 1 / 4$ of hypodensities without enchancement. ${ }^{36}$ Brain MRI delineates more lesions, but normal studies may occur. Abnormalities include T2 hyperintensities in the centre of the pons, the tegmentum and the corticospinal tracts. ${ }^{5} 37$ Isolated extrapontine demyelination may occur in $\sim 10 \%$ of patients (ie, basal ganglia, thalami, lateral geniculate body, cerebellum and cerebral cortex ${ }^{5} 3637$ ), or may occur together with CPM. Lesions can occasionally be seen in the thalamus, putamen, caudate nucleus, midbrain and pons on FLAIR and T2-weighted and T1-weighted imaging-extrapontine lesions seen in $>40 \% .^{38}$ Early examination with diffusionweighted MRI reveals changes of in water diffusion $<1$ day after first symptoms. ${ }^{37}$ Regional hyperperfusion may be delineated during recovery using SPECT, with decreases in the striatal dopamine transporter in extrapontine myelinolysis. ${ }^{39}$

The severity of demyelination expressed by the volume of MRI signal abnormality, however, may not correspond to the degree of clinical deficit, or return of function, as it is not associated with poor outcome, ${ }^{36} 38$ and residual signal changes may persist despite complete clinical recovery. Table 5 summarises frequent neuroimaging patterns. 
Wernicke's encephalopathy

Pathophysiology: Wernicke's encephalopathy is caused by a thiamine deficiency in alcohol abuse, malabsorption, poor nutrition, increased metabolism or iatrogenic elimination by haemodialysis. Thiamine deficiency may cause an osmotic imbalance with consequent perineural oedema and neuronal swelling. ${ }^{40}$ Periventricular lesions may be explained by a focal high rate of a thiamine-dependent metabolism. ${ }^{41}$

Neuroimaging (see online supplementary table S3, figure 2A,B): Cerebral CT and MRI changes may show hypodensities, or hyperintensities in the midbrain, diencephalon and periventricular areas enhance. ${ }^{42} 43$ DWI changes include hyperintensities on T2-weighted, and hypointensities on T1-weighted sequences near the third ventricle, mammillary bodies, the cerebral aqueduct and thalami. ${ }^{43}$ Chronically, there may be focal atrophy, especially of the mammillary bodies. ${ }^{44}$ MRS reveals a decrease in aspartate and increase in lactate in involved areas. $^{45}$ There are no SPECT or PET studies.

Many of the abnormalities regress with treatment, but once there is involvement of the cortex on brain MRI, prognosis is poor in up to $100 \% .{ }^{46}$ Frequent neuroimaging patterns are summarised in table 6 .
Posterior reversible encephalopathy

Pathophysiology: Posterior reversible encephalopathy syndrome (PRES) - a rare and potentially reversible neurotoxic state with a breakdown of the cerebral circulatory autoregulation ${ }^{47}$-is often associated with (pre-)eclampsia and less frequently with other pathological conditions, such as hypertension, sepsis, Guillain-Barré syndrome, autoimmune connective tissue disorders, chemotherapy and organ transplantation. Precursors include vasospasm, vasculitis and serum hyperviscosity. ${ }^{47}$ There are different hypotheses regarding the pathogenesis of PRES: (1) capillary vessels secondarily injured by severe arterial hypertension leading to hyperperfusion and cerebral oedema, and (2) hypertension-related reflexive vasoconstriction with secondary brain hypoperfusion and subsequent oedema. ${ }^{48}$

Neuroimaging (see online supplementary table S3, figure 3A): Cerebral CT reveals areas of hypodensity, but may be nonspecific. MRI can demonstrate subcortical and cortical oedema in watershed zones, posterior cerebral artery perfusion areas in parietal and occipital regions, and sometimes also in frontal and temporal watershed zones. Cerebellar changes are less frequent. MRI shows changes that involve several vascular territories in the form of T1 and T2 prolongation, ${ }^{5}$ with alterations in the basal ganglia, thalamus, brainstem and centrum semiovale. ${ }^{49}$ In

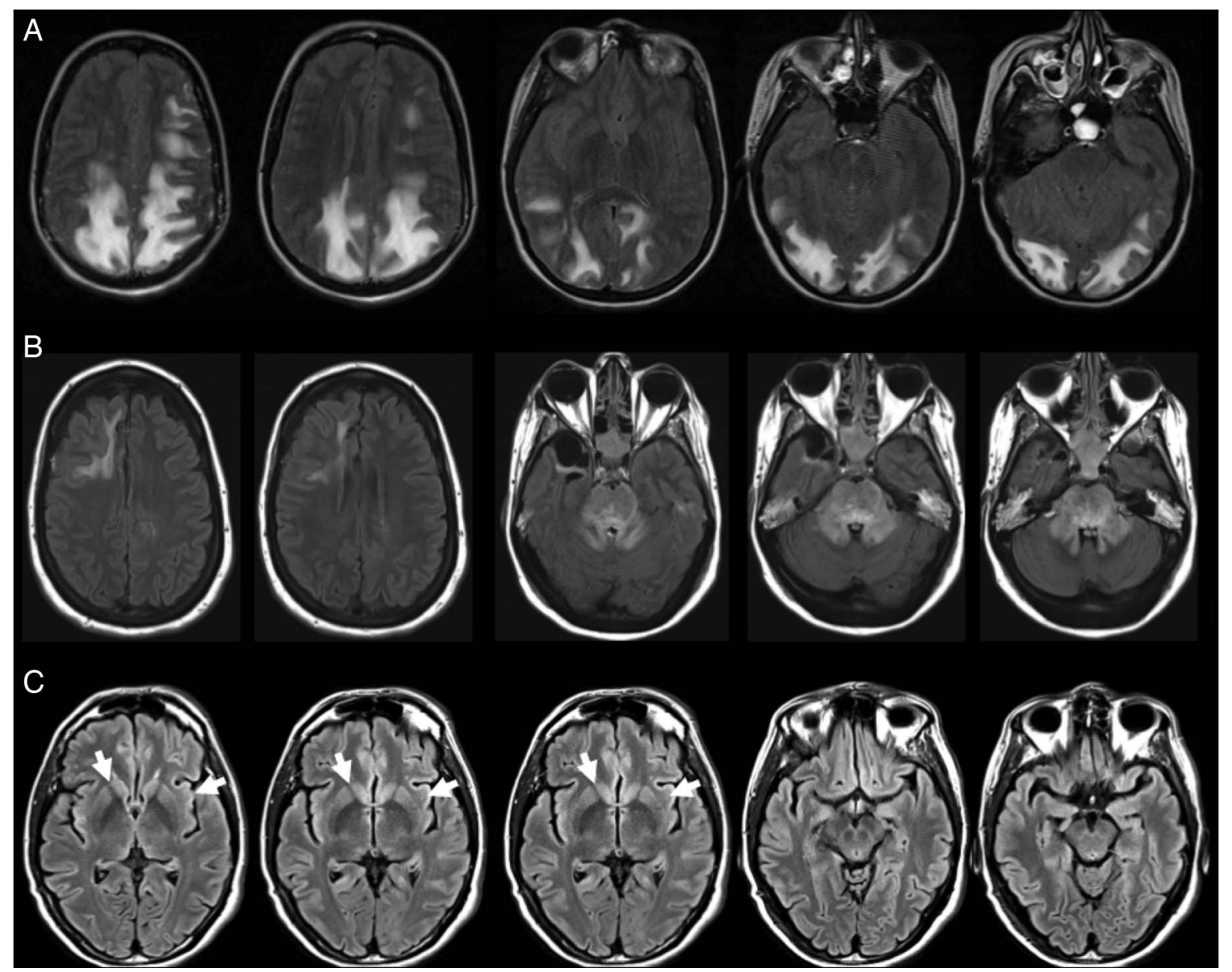

Figure $3 \mathrm{MRI}$ in specific acute encephalopathy syndromes. Serial axial fluid-attenuated inversion recovery sequences (on 1.5-T MRI). (A) Posterior reversible encephalopathy syndrome with widespread confluent subcortical hyperintensities in the posterior circulation; and (B) acute disseminated encephalomyelitis with subcortical hyperintensities in the right frontal lobe ( 3 images on the right), in the brain stem and in the cerebellar peduncles ( 3 images on the left); and (C) Creutzfeldt-Jakob disease (arrows mark hyperintense areas of the cortex-the 'cortical ribbon sign' (right arrow) - , putamen and caudate head isointense with the cortical grey (left arrows)). 
76 patients with suspected PRES, MRI showed intraparenchymal haemorrhages in only $17 \%{ }^{49}$ MRS reveals decreased $\mathrm{N}$-acetyl-aspartate/choline and $\mathrm{N}$-acetyl-aspartate/creatinine ratios outside the areas of oedema. ${ }^{50}$ Vasoconstriction may appear on cerebral CT, MR angiography or SPECT.

Neuroimaging changes may regress during days to weeks. The extent of MRI abnormalities correlates with outcome with T2/ DWI scores of non-survivors being significantly higher than those of patients who recovered. ${ }^{51}$ The predictive value of MRI findings is given in table 7 . Frequent neuroimaging patterns are summarised in table 6 .

\section{Autoimmune-induced encephalopathy}

Acute disseminated encephalomyelitis and the Marburg variant Pathophysiology: Acute disseminated encephalomyelitis (ADEM) is a non-specific term referring to acute CNS inflammation that is postinfectious or parainfectious, postvaccinal or of unknown origin. Exact mechanisms are not understood. The disseminated inflammation is usually monophasic and neurological symptoms result from involvement of cortical and subcortical structures, as well as the spinal cord. There is growing evidence that supports an autoimmune mechanism, as immunological changes, such as increased serum IgA, circulating immune complexes, and a decrease of $\mathrm{T}$ and $\mathrm{B}$ lymphocytes are frequently found. The exact mechanisms of the Marburg variant of multiple sclerosis, which subsequently follows an aggressive course of demyelination and leads to death within 1 year, remain unclear. ${ }^{52}$ There may be severe myelin and axon destruction with prominent tissue necrosis and macrophage infiltration.

Neuroimaging (figure 3B): Cerebral CT and MRI are often normal, ${ }^{53}$ but abnormalities can occur with contrast enhancement on T2-weighted images and FLAIR sequences in the acute phase. ${ }^{53}$ Most patients have multiple brain lesions in the deep and subcortical white matter characteristic of demyelination, and up to $1 / 3$ have lesions in the brainstem and spinal cord. ${ }^{53} 54$ DWI shows variable abnormalities depending on the stage of the disease. In the first week (ie, acute phase) DWI reveals a restricted diffusion, and later (ie, subacute phase) diffusion increases. $^{55}$ Small MRS studies showed a reduction of $\mathrm{N}$-acetyl-aspartate in regions corresponding to the areas of high T2 signal intensity in the subacute phase. ${ }^{55}$ Haemorrhagic demyelinating lesions are mostly seen in the hyperacute ADEM variants. SPECT and PET have not been studied extensively.

Imaging often improves, although lesions may persist. Radiographically, the lesions of the Marburg variant demonstrate significant mass effect and oedema but the patterns may overlap with findings in ADEM. Hence, the Marburg variant may be best considered the most extreme type of demyelinating diseases and distinguishing it from other fulminant variants, such as ADEM may be difficult. ${ }^{52}$ Frequent neuroimaging patterns of ADEM are summarised in table 8.

\section{Autoimmune and paraneoplastic limbic encephalitis}

Pathophysiology: Paraneoplastic and autoimmune limbic encephalitis (LE) can develop acutely or subacutely and may represent limbic status epilepticus. Most paraneoplastic LE are associated with autoantibodies and cytotoxic $\mathrm{T}$ cells that target the onconeuronal proteins. Among the many different types of autoimmune and paraneoplastic LE, we focus on NMDA receptor antibody-induced LE and Hashimoto's encephalopathy in the following section.

Anti-NMDA receptor antibody-induced LE is mostly associated with prolonged encephalopathy, with progressive movement disorders, seizures, changes of behaviour, rapid dementia and confusional states. ${ }^{56}$ Hashimoto's encephalopathy or steroid-responsive encephalitis with autoimmune thyroiditis (SREAT) - another specific type of LE_can be associated with Hashimoto's thyroiditis. Some believe that the association is spurious. SREAT is characterised by confusion, psychosis, memory loss, gait problems and subacute or chronic encephalopathy with cognitive decline and even coma. Neuropathological examinations show perivascular inflammatory infiltration, ${ }^{57}$ however, the exact mechanisms remain unclear.

Neuroimaging: In paraneoplastic LE, cerebral CT is often normal, while the MRI is more sensitive, with contrast enhancing mesial temporal T2 and FLAIR hyperintensities in $>50 \%$. Subcortical regions, the cerebellum or brainstem may be involved. ${ }^{58}$ A diagnosis is made in almost $80 \%$ of patients based on clinical features, presence of paraneoplastic antibodies and the MRI patterns aforementioned. MRI can be used to rule out other aetiologies. ${ }^{59}$ PET may reveal increased metabolism mesial temporal. $^{60}$

In NMDA receptor-mediated LE, FLAIR and T2 hyperintensity signal changes typically involve temporal regions, and sometimes extratemporal areas. MRI is used to exclude other causes of acute encephalopathy.

In SREAT, MRI abnormalities are rare (in about $1 / 3$ of patients). ${ }^{61}$ However, white matter changes can occur and are atypical, or can resemble ADEM (aforementioned). ${ }^{62}$ Other reports include hippocampal and multifocal hyperintensities on T2, FLAIR and DWI with corresponding hypointensities on T1-weighted sequences. ${ }^{63}$ MRS reveals decreased $\mathrm{N}$-acetyl-aspartate, myoinositol peaks, elevations in lipid, lactate, glutamate/glutamine and choline peaks. Some cases show regression of these pathological signals after high doses of corticosteroids. ${ }^{64}$ On SPECT, decreased tracer uptake in the right striatum ${ }^{65}$ and global hypoperfusion of the whole cerebral cortex are reported. ${ }^{66}$ Outcome is mostly favourable. Frequent neuroimaging patterns are summarised in table 8. Data from other rarer autoimmune LE are not presented.

\section{Herpes simplex encephalitis}

Pathophysiology: In immunocompetent adults, more than $90 \%$ of HSV encephalitis (HSVE) cases are caused by HSV type $1 .{ }^{67}$ Whether HSVE is due to primary infection or viral reactivation is uncertain. The pathomechanism of HSV entry into the brain is still undetermined and could be due to viral genome reactivation in the natural reservoir in the trigeminal ganglion with axonal spread via the trigeminal nerve into the CNS, in situ reactivation of the latent virus from CNS tissue, or primary infection of the CNS. Pathways for entry of HSV into the brain include both the olfactory and the trigeminal nerves. Although HSVE is not considered a disorder of the immunocompromised, cases are described in the context of bone marrow transplantation, HIV infection, and in association with an impaired cellular interferon $\alpha / \beta$ and $\lambda$ antiviral responses. ${ }^{68}$

Neuroimaging: Cerebral CT is usually normal within the first week but can characteristically show reduced attenuation in the temporal lobes later in the course. CT has therefore been substituted by MRI, which is more sensitive, showing FLAIR, T2 and DWI hyperintensities in the medial temporal lobes, the orbital surface of the frontal lobes, the insular cortex, the angular gyrus and in the insulas early in the course in $>90 \%$ of patients. ${ }^{69}$ Abnormal areas may show enhancement with gadolinium. Midline shift may be present with large cerebral oedema. Rarely, brain MRI can be normal. ${ }^{70}$ Differences between imaging appearance of LE and herpes simplex encephalitis are 
Table 7 Most consistent and pathognomonic neuroimaging patterns in the literature and their predictive value (data from the online supplementary tables $\mathrm{S1-S3)}$

Posterior reversible encephalopathy

CT Hypodensities in the parieto-occipital subcortical white matter and cerebellum with increased cerebral blood volume, blood flow, and reduced time to peak mainly in the posterior vascular distribution

MRI - T2, FLAIR and DWI hyperintensities in the posterior circulation areas and, less frequent, in the anterior circulation structures. ADC values in areas of abnormal T2 signal are high

- Contrast enhancement, restrictions on DWI and ADC

- Decrease in $\mathrm{N}$-acetyl-aspartate on MRS in patients with normal MRI or reversible MRI changes and only minimal elevation of choline

Acute disseminated encephalomyelitis

CT Usually normal

MRI $\quad$ T2 and FLAIR with multiple brain lesions in the deep and subcortical white matter and in $1 / 3$ in the brainstem and spinal cord characteristic of demyelination with contrast enhancement

- In the first week (acute phase) DWI with restricted diffusion, and later (subacute phase) with increased diffusion

- Decreased $\mathrm{N}$-acetyl-aspartate in regions with $\mathrm{T} 2$ hyperintensities in the subacute phase on MRS

Paraneoplastic limbic encephalitis

CT Usually normal

MRI $\quad \mathrm{T} 2$ and FLAIR hyperintensities with mesial temporal contrast enhancement in $>50 \%$ and/or atrophy

- Subcortical regions, the cerebellum or brainstem may be involved

PET PET may reveal increased metabolism mesial temporal

Autoimmune limbic encephalitis

CT Usually normal

MRI In NMDAR-antibody-mediated limbic encephalitis, MRI is mostly (>50\%) normal but can show T2 and FLAIR hyperintensities temporal and rarely extratemporal. MRI is used to exclude other causes of encephalopathy

- In SREAT, T2 and FLAIR can rarely resemble acute demyelinating encephalomyelitis or show hippocampal or multifocal hyperintensities

- In SREAT, MRS shows decreased $\mathrm{N}$-acetyl-aspartate, myoinositol peaks, elevations in lipid, lactate, glutamate/glutamine and choline peaks support inflammation

SPECT In NMDAR-antibody-mediated limbic encephalitis, abnormal multifocal cerebral blood flow

- In SREAT, decreased tracer uptake in the striatum and global cortical hypoperfusion

Herpes simplex encephalitis

CT Usually normal, but can characteristically show reduced attenuation in the temporal lobes after the first week of the disease

MRI $\quad$ T2, FLAIR and DWI hyperintensities in the medial temporal lobes, the orbital surface of the frontal lobes, the insular cortex, the angular gyrus and in the insulas early in the course

- Abnormal areas may show enhancement with gadolinium

- Midline shift may be present with large cerebral oedema

SPECT Increased tracer accumulation which reflects hyperperfusion possibly earlier than pathological signals appear on MRI

Susac's syndrome

CT Does not reveal any of the specific structural abnormalities, but can demonstrate foci of subtle low attenuation in the corpus callosum

MRI T2 and FLAIR hyperintensities in the corpus callosum, which is always involved

- Any part of the corpus callosum can be involved, but predominately the central fibres are showing microinfarcts that are typically small but may sometimes be large

- Foci in the corpus callosum may enhance following gadolinium administration and there can be restricted diffusion with corresponding low-signal intensity on the ADC map

- Spinal cord involvement is rare but exists

- Subsequently, central callosal holes arise

PET Marked hypometabolism in the frontal, parietal and temporal lobes-an unspecific pattern that can be mistaken as ADEM

Creutzfeldt-Jakob disease

CT Non-specific generalised cortical and subcortical atrophy in the later phases of disease
No clear evidence

More extensive T2 signal abnormalities were seen with poor outcome

No clear evidence

No clear evidence

No clear evidence

No clear evidence

No clear evidence

No clear evidence

No clear evidence

No clear evidence

Lesions on $\mathrm{CT}$ are predictive of prolonged course of disease

The extent of brain involvement is an independent risk factor for poor prognosis

No clear evidence

No clear evidence

No clear evidence

No clear evidence

No clear evidence 
Table 7 Continued

\begin{tabular}{|c|c|c|}
\hline Imaging & Patterns & Predictive value for poor outcome \\
\hline MRI & $\begin{array}{l}\text { T2 and FLAIR hyperintensities in the cerebral cortex and lesions in the putamen } \\
\text { and caudate head isointense to cortex and lesions in the putamen and caudate } \\
\text { head isointense to cortical grey } \\
\text { Less frequently, hyperintensity can be detected in the globus pallidus, thalamus, } \\
\text { the deep white matter, and the cerebral and cerebellar cortex. Laminar lesions } \\
\text { may be observed in the cerebral cortex and cerebellum } \\
\text { DWI is most sensitive in early stages uncovering the altered diffusion in the } \\
\text { regions aforementioned } \\
\text { In vCJD symmetrical hyperintensities of the pulvinar thalami (relative to the } \\
\text { cortex and especially the anterior part of the putamen) are characteristic and } \\
\text { known as the 'pulvinar sign' } \\
\text { Decreased } N \text {-acetyl-aspartate and slightly increased levels of myoinositol in the } \\
\text { striatum and the insular cortex on MRS }\end{array}$ & $\begin{array}{l}\text { Patients with cortical plus basal ganglia hyperintensity have shorter } \\
\text { interval from symptom onset to akinetic mutism than those with isolated } \\
\text { cortical ribbon hyperintensity }\end{array}$ \\
\hline PET & Hypometabolism in the cerebral cortex and the basal ganglia & No clear evidence \\
\hline SPECT & Hypoperfusion in the cerebral cortex & No clear evidence \\
\hline
\end{tabular}

summarised in table 7. Studies of MRS, SPECT and PET are lacking. Case series report an increased tracer accumulation in SPECT, which does not reflect blood-brain barrier disruption or

Table 8 Frequent neuroimaging patterns in autoimmune-induced encephalopathy

\begin{tabular}{ll}
\hline Imaging & Patterns \\
\hline Acute disseminated encephalomyelitis \\
CT
\end{tabular}

ADEM, acute disseminated encephalomyelitis; DWI, diffusion-weighted imaging; FLAIR, fluid-attenuated inversion recovery; MRS, MR spectroscopy; NMDAR, $\mathrm{N}$-methyl-D-aspartate receptor; SPECT, single-photon emission CT; SREAT, steroid-responsive encephalitis with autoimmune thyroiditis. inflammatory oedema, but rather hyperperfusion possibly earlier than pathological signals appear on MRI. ${ }^{71}$

While small investigations regarding the predictive value of cerebral CT showed that lesions on CT are predictive of prolonged course of the disease, ${ }^{72-74}$ a study of 106 patients with HSVE revealed that the extent of brain involvement on MRI at admission is an independent risk factor for poor prognosis. ${ }^{69}$ Frequent neuroimaging patterns are summarised in table 9 .

\section{Susac's syndrome-related encephalopathy}

Pathophysiology: Susac's syndrome is a rare disease that is characterised by the clinical triad of encephalopathy, branch retinal artery occlusion, and sensorineural hearing loss. ${ }^{75}$ The pathogenesis of Susac's syndrome remains unclear. Different mechanistic hypothesis have been implicated, such as immune mechanisms, vasospastic phenomena, coagulopathy and viral infection. However, these hypotheses remain unproven. The diagnosis is mainly based on brain MRI, retinal fluorescein angiography and audiometry.

Neuroimaging: Cerebral CT mostly does not reveal any of the specific structural abnormalities, but can demonstrate foci of subtle low attenuation in the corpus callosum. ${ }^{76} \mathrm{~T} 2$ and FLAIR sequences of brain MRI are very sensitive detecting signal changes in the corpus callosum, which is always involved. Any part of the corpus callosum can be affected, but predominately the central fibres show microinfarcts that are typically small. ${ }^{76}$ Foci in the corpus callosum often mildly enhance following gadolinium administration and

Table 9 Neuroimaging patterns in herpes simplex encephalitis

\section{Imaging Patterns}

Herpes simplex encephalitis

CT Usually normal, but can characteristically show reduced attenuation in the temporal lobes after the first week of the disease

MRI T2, FLAIR and DWI hyperintensities in the medial temporal lobes, the orbital surface of the frontal lobes, the insular cortex, the angular gyrus, and in the insulas early in the course

- Abnormal areas may show enhancement with gadolinium

- Midline shift may be present with large cerebral oedema

SPECT Increased tracer accumulation which reflects hyperperfusion possibly earlier than pathological signals appear on MRI

DWI, diffusion-weighted imaging; FLAIR, fluid-attenuated inversion recovery; SPECT, single-photon emission CT. 
Table 10 Neuroimaging patterns in encephalopathy caused by Susac's syndrome

Imaging Patterns
Susac's syndrome-related encephalopathy
CT Does not reveal any of the specific structural abnormalities, but can
demonstrated foci of subtle low attenuation in the corpus callosum
MRI T2 and FLAIR hyperintensities in the corpus callosum, which is
always involved
- Any part of the corpus callosum can be involved, but
predominately the central fibres are showing microinfarcts that are
typically small but may sometimes be large
- Foci in the corpus callosum may enhance following gadolinium
administration and there can be restricted diffusion with
corresponding low-signal intensity on the ADC map
- Spinal cord involvement is rare but exists
MEtsequently, central callosal holes arise
Marked hypometabolism in the frontal, parietal and temporal lobes
- an unspecific pattern that can be mistaken as ADEM

$A D C$, apparent diffusion coefficient; ADEM, acute disseminated encephalomyelitis; FLAIR, fluid-attenuated inversion recovery; PET, positron emission tomography.

there can be restricted diffusion with corresponding low-signal intensity on the ADC map. ${ }^{76}$ These are followed by central callosal holes which may be pathognomonic. ${ }^{77}$ Aside from these typical findings, any part of the brain can be affected, but the white matter is most frequently involved. ${ }^{78}$ Few articles of MRS report no further useful information in this context and studies of SPECT are lacking. Case reports of brain FDG-PET show marked widespread hypometabolism in the frontal, parietal and temporal lobes-an unspecific pattern that can be mistaken as ADEM. ${ }^{79}$ Frequent neuroimaging patterns are summarised in table 10.

\section{Creutzfeldt-Jakob disease}

Pathophysiology: Prion diseases are transmissible neurodegenerative diseases with long incubation periods. Once clinical symptoms appear, the fatal course of the disease is inexorable. From the currently known human prion diseases kuru,

Table 11 Neuroimaging patterns in encephalopathy caused by Creutzfeldt-Jakob disease

\begin{tabular}{ll}
\hline Imaging & Patterns \\
\hline Creutzfeldt-Jakob disease-related encephalopathy \\
CT $\quad$ Non-specific generalised cortical and subcortical atrophy in the later \\
phases of disease \\
MRI $\quad$ T2 and FLAIR hyperintensities in the cerebral cortex and lesions in \\
the putamen and caudate head isointense to cortex lesions in the \\
putamen and caudate head isointense to cortical grey \\
Less frequently, hyperintensity can be detected in the globus \\
pallidus, thalamus, the deep white matter, and the cerebral and \\
cerebellar cortex. Laminar lesions may be observed in the cerebral \\
cortex and cerebellum \\
DWI is most sensitive in early stages uncovering the altered \\
diffusion in the regions aforementioned \\
In vCJD symmetrical hyperintensities of the pulvinar thalami \\
(relative to the cortex and especially the anterior part of the \\
putamen) are characteristic and known as the 'pulvinar sign' \\
Decreased $N$-acetyl-aspartate and slightly increased levels of \\
myoinositol in the striatum and the insular cortex on MRS \\
SPECT Hypoperfusion in the cerebral cortex \\
HET
\end{tabular}

DWI, diffusion-weighted imaging; FLAIR, fluid-attenuated inversion recovery; MRS, MR spectroscopy; PET, positron emission tomography; SPECT, single-photon emission CT; vCJD, variant Creutzfeldt-Jakob disease.
Creutzfeldt-Jakob disease (CJD), variant CJD (vCJD), Gerstmann-Sträussler-Scheinker syndrome, and fatal familial insomnia, CJD is the most prevalent $(>90 \%$ of the prion diseases). ${ }^{80}$ In spongiform encephalopathies, prions can induce a pathological refolding of native proteins-a self-sustaining process leading to insoluble proteins that disrupt neuronal function and cause cell death. Neuronal loss, glial proliferation, absence of inflammation and small vacuoles within the neuropil, are common pathological characteristics.

Neuroimaging (figure 3C): While cerebral CT shows nonspecific generalised cortical and subcortical atrophy in the later phases of disease, ${ }^{81} \mathrm{MRI}$ is more helpful. Typically, the putamen and caudate head are isointense to cortical grey on T2 and FLAIR. ${ }^{82}$ Less frequently, hyperintensity can be detected in the globus pallidus, thalamus, the deep white matter, and the cerebral and cerebellar cortex. Laminar lesions may be observed in the cerebral cortex and cerebellum. Proton density and DWI are more sensitive, especially for cortical lesions. DWI is most sensitive in early stages of CJD and can uncover brain abnormalities before the onset of clinical symptoms, such as myoclonus. ${ }^{83}$ One study of MRI in 90 patients demonstrated that the combination of FLAIR and DWI hyperintensities and restricted diffusion could reliably differentiate between CJD and other rapidly progressive dementia with a high sensitivity (94\%) and specificity $(100 \%) .{ }^{84}$ However, a study of more than 1000 patients with pathologically confirmed CJD found that MRI interpretation had a sensitivity of $46 \%$ when obtained at least 6 months after CJD onset, requiring some caution when interpreting MRI. ${ }^{85}$ MRI abnormalities may vary with the clinical syndrome and molecular subtypes. In late or terminal stages of CJD prominent generalised cortical and subcortical atrophy is seen. In vCJD symmetrical hyperintensities of the pulvinar thalami (relative to the cortex and especially the anterior part of the putamen) are characteristic and known as the 'putaminal sign'. ${ }^{86}$ Descriptions of abnormal MRS, PET and SPECT are limited to single case reports of patients with sporadic CJD and have not been further evaluated.

Patients with cortical plus basal ganglia hyperintensity have shorter interval from symptom onset to akinetic mutism than those with isolated cortical ribbon hyperintensity. ${ }^{87}$ Frequent neuroimaging patterns are summarised in table 11.

\section{Toxic encephalopathies}

Another important group of acute encephalopathies, the toxic encephalopathies resulting from exogenous substances, may appear with typical or unspecific imaging patterns. Rather typical imaging patterns, as putaminal lesions may be found on MRI in methanol intoxication ${ }^{88} 89$ and a diffuse swelling and bipallidal haemorrhages in ethylene glycol poisoning. ${ }^{90} \mathrm{~A}$ comprehensive summary of all toxic encephalopathies is beyond the scope of this review.

\section{SUMMARY}

Cerebral imaging constitutes an important component of diagnosis, management and prognosis of acute encephalopathy. Its respective contribution is dominated by rapid exclusion of acute cerebral lesions and further varies greatly depending on the underlying aetiology and the range of possible differential diagnoses. For these disorders, cerebral CT has been well studied, but is largely insensitive, while brain MRI and especially DWI have been widely reported and appear to be the most helpful in the evaluation of acute encephalopathies. MRS may provide supplementary biochemical information and determines spectral changes in the affected brain tissue. PET and SPECT have their 
use in delineating areas of high or low metabolic activity or cerebral blood flow (eg, with LE or vasospasm). However, in most of the compiled types of acute encephalopathy, publications of MRS, PET and SPECT are limited to case reports and small case series only providing anecdotal evidence of their usefulness and sensitivity, not to mention that the observations are often heavily technique dependent. Most consistent and pathognomonic neuroimaging patterns and their prognostic are compiled in tables 1 and 7. To date, the prognostic value of specific imaging patterns has been quantified especially for MRI in patients with HIE, hypoglycaemic encephalopathy and PRES while in other encephalopathies larger studies are needed to provide such critical information. Many of the imaging changes are non-specific, and the clinician must judiciously use cerebral imaging to supplement clinical impressions, relevant blood, urine or antibody tests, EEG and occasionally body PET. Findings can then be used to guide treatment and occasionally point to outcome in these morbid conditions.

Contributors RS and PWK conceived and planned the work, acquired, analysed and interpreted the data, and wrote a first draft of the manuscript. Both the authors approved the final submitted version.

Competing interests RS is supported by the Research Funds of the University of Basel, the Scientific Society Basel, and the Gottfried Julia Bangerter-Rhyner Foundation. PWK reports grants from Qatar National Research Foundation, personal fees from Royalties Wiley, Demos for books on epilepsy and EEG; honoraria for international congresses and grand rounds Europe and North America; travel to give lectures Europe and North America; consulting fee for EEG reading Esai Pharma; Expert testimony on $\mathrm{qEEG}$ in the courtroom, non-financial support from non-paid board member ACNS and $A B C N$, outside the submitted work.

Provenance and peer review Not commissioned; externally peer reviewed.

\section{REFERENCES}

1 American Psychiatric Association. Diagnostic and statistical manual of mental disorders: DSM-IV. Task Force on DSM-IV, editor. American Psychiatric Publication, 2000.

2 Choi DW. Excitotoxic cell death. J Neurobiol 1992;23:1261-76.

3 Kaplan PW, Rossetti AO. EEG patterns and imaging correlations in encephalopathy: encephalopathy part II. J Clin Neurophysiol 2011;28:233-51.

4 Huang BY, Castillo M. Hypoxic-ischemic brain injury: imaging findings from birth to adulthood. Radiographics 2008;28:417-39; quiz 617

5 Bathla G, Hegde AN. MRI and CT appearances in metabolic encephalopathies due to systemic diseases in adults. Clin Radiol 2013;68:545-54.

6 Arbelaez A, Castillo M, Mukherji SK. Diffusion-weighted MR imaging of global cerebral anoxia. AJNR Am J Neuroradiol 1999;20:999-1007.

7 Wijman CA, Mlynash M, Caulfield AF, et al. Prognostic value of brain diffusion-weighted imaging after cardiac arrest. Ann Neurol 2009;65:394-402

8 Wijdicks EF, Campeau NG, Miller GM. MR imaging in comatose survivors of cardiac resuscitation. AJNR Am J Neuroradiol 2001;22:1561-5.

9 Stevens RD, Sutter R. Prognosis in severe brain injury. Crit Care Med 2013;41:1104-23.

10 Fugate JE, Wijdicks EF, Mandrekar J, et al. Predictors of neurologic outcome in hypothermia after cardiac arrest. Ann Neurol 2010;68:907-14.

11 Choi SP, Park KN, Park HK, et al. Diffusion-weighted magnetic resonance imaging for predicting the clinical outcome of comatose survivors after cardiac arrest: a cohort study. Crit Care 2010;14:R17.

12 Cronberg $T$, Rundgren $M$, Westhall $E$, et al. Neuron-specific enolase correlates with other prognostic markers after cardiac arrest. Neurology 2011;77:623-30.

13 Kreis $R$, Arcinue E, Ernst $T$, et al. Hypoxic encephalopathy after near-drowning studied by quantitative $1 \mathrm{H}$-magnetic resonance spectroscopy. J Clin Invest 1996;97:1142-54.

14 Sharshar T, Annane D, de la Grandmaison GL, et al. The neuropathology of septic shock. Brain Pathol 2004;14:21-33.

15 Piazza 0 , Cotena S, De Robertis E, et al. Sepsis associated encephalopathy studied by MRI and cerebral spinal fluid S100B measurement. Neurochem Res 2009;34:1289-92.

16 Sharshar T, Carlier R, Bernard F, et al. Brain lesions in septic shock: a magnetic resonance imaging study. Intensive Care Med 2007:33:798-806.

17 Park JH, Kim HJ, Kim SM. Acute chorea with bilateral basal ganglia lesions in diabetic uremia. Can J Neurol Sci 2007:34:248-50.

18 Wang HC, Brown P, Lees AJ. Acute movement disorders with bilateral basal ganglia lesions in uremia. Mov Disord 1998;13:952-7.
19 Kang E, Jeon SJ, Choi SS. Uremic encephalopathy with atypical magnetic resonance features on diffusion-weighted images. Korean J Radiol 2012;13:808-11.

20 Sheu YL, Cheng SJ, Chen YM, et al. The syndrome of bilateral basal ganglia lesions in diabetic uremic patients presenting with a relapsing and remitting course: a case report. Acta Neurol Taiwan 2007;16:226-30.

21 Dicuonzo F, Di Fede R, Salvati A, et al. Acute extrapyramidal disorder with bilateral reversible basal ganglia lesions in a diabetic uremic patient: diffusion-weighted imaging and spectroscopy findings. J Neurol Sci 2010;293:119-21.

22 Wang HC, Hsu JL, Shen YY. Acute bilateral basal ganglia lesions in patients with diabetic uremia: an FDG-PET study. Clin Nucl Med 2004;29:475-8.

23 Rimar D, Bitterman H. Hyperammonemic coma: beyond hepatic encephalopathy. South Med J 2008:101:467-8.

24 Takanashi J, Barkovich AJ, Cheng SF, et al. Brain MR imaging in acute hyperammonemic encephalopathy arising from late-onset ornithine transcarbamylase deficiency. AJNR Am J Neuroradiol 2003;24:390-3.

25 Rovira A, Alonso J, Cordoba J. MR imaging findings in hepatic encephalopathy. AJNR Am J Neuroradiol 2008;29:1612-21.

26 Nomiyama K, Tsuji H, Ikeda K, et al. [Positron emission tomography (PET) before and after treatment of hyperammonemia in a patient with decompensated liver cirrhosis]. Fukuoka Igaku Zasshi 1991;82:521-7.

27 McKinney AM, Lohman BD, Sarikaya B, et al. Acute hepatic encephalopathy: diffusion-weighted and fluid-attenuated inversion recovery findings, and correlation with plasma ammonia level and clinical outcome. AJNR Am J Neuroradiol 2010:31:1471-9.

28 Kohira I, Matsuo E, Shiro Y, et al. Hepatic encephalopathy with increased cerebral blood flow in SPECT and MRI abnormalities in the basal ganglia. Jpn J Psychiatry Neurol 1994;48:33-6.

29 Weissenborn K, Berding G, Kostler H. Altered striatal dopamine D2 receptor density and dopamine transport in a patient with hepatic encephalopathy. Metab Brain Dis 2000;15:173-8.

30 Nakagawa Y, Matsumura K, Iwasa M, et al. Single photon emission computed tomography and statistical parametric mapping analysis in cirrhotic patients with and without minimal hepatic encephalopathy. Ann Nucl Med 2004;18: 123-9.

31 Fujioka M, Okuchi $\mathrm{K}$, Hiramatsu Kl, et al. Specific changes in human brain after hypoglycemic injury. Stroke 1997;28:584-7

32 Lim CC, Gan R, Chan CL, et al. Severe hypoglycemia associated with an illegal sexual enhancement product adulterated with glibenclamide: MR imaging findings. Radiology 2009;250:193-201.

$33 \mathrm{Ma}$ JH, Kim YJ, Yoo WJ, et al. MR imaging of hypoglycemic encephalopathy: lesion distribution and prognosis prediction by diffusion-weighted imaging. Neuroradiology 2009:51:641-9.

34 Lai $\mathrm{PH}$, Tien RD, Chang MH, et al. Chorea-ballismus with nonketotic hyperglycemia in primary diabetes mellitus. AJNR Am J Neuroradiol 1996;17:1057-64.

35 Strange $K$, Jackson PS. Swelling-activated organic osmolyte efflux: a new role for anion channels. Kidney Int 1995;48:994-1003.

36 Menger $\mathrm{H}$, Jorg J. Outcome of central pontine and extrapontine myelinolysis ( $\mathrm{n}=$ 44). J Neurol 1999:246:700-5.

37 Ruzek KA, Campeau NG, Miller GM. Early diagnosis of central pontine myelinolysis with diffusion-weighted imaging. AJNR Am J Neuroradiol 2004;25:210-13.

38 Graff-Radford J, Fugate JE, Kaufmann TJ, et al. Clinical and radiologic correlations of central pontine myelinolysis syndrome. Mayo Clin Proc 2011;86: 1063-7.

$39 \mathrm{Kim}$ JS, Lee KS, Han SR, et al. Decreased striatal dopamine transporter binding in a patient with extrapontine myelinolysis. Mov Disord 2003;18:342-5.

40 Zuccoli G, Gallucci M, Capellades J, et al. Wernicke encephalopathy: MR findings at clinical presentation in twenty-six alcoholic and nonalcoholic patients. AJNR Am J Neuroradiol 2007;28:1328-31.

41 Victor M, Adams RD, Collins GH. The Wernicke-Korsakoff syndrome. A clinical and pathological study of 245 patients, 82 with post-mortem examinations. Contemp Neurol Ser 1971;7:1-206.

42 Antunez E, Estruch R, Cardenal C, et al. Usefulness of CT and MR imaging in the diagnosis of acute Wernicke's encephalopathy. AJR Am J Roentgenol 1998:171:1131-7.

43 Gallucci M, Bozzao A, Splendiani A, et al. Wernicke encephalopathy: MR findings in five patients. AJNR Am J Neuroradiol 1990;11:887-92.

44 Park SH, Kim M, Na DL, et al. Magnetic resonance reflects the pathological evolution of Wernicke encephalopathy. J Neuroimaging 2001;11:406-11.

45 Murata T, Fujito T, Kimura H, et al. Serial MRI and (1)H-MRS of Wernicke's encephalopathy: report of a case with remarkable cerebellar lesions on MRI. Psychiatry Res 2001;108:49-55.

46 Fei GQ, Zhong C, Jin L, et al. Clinical characteristics and MR imaging features of nonalcoholic Wernicke encephalopathy. AJNR Am J Neuroradiol 2008;29:164-9.

47 Hinchey J, Chaves C, Appignani B, et al. A reversible posterior leukoencephalopathy syndrome. N Engl J Med 1996:334:494-500.

48 Bartynski WS. Posterior reversible encephalopathy syndrome, part 2: controversies surrounding pathophysiology of vasogenic edema. AJNR Am J Neuroradiol 2008;29:1043-9. 
49 McKinney AM, Short J, Truwit CL, et al. Posterior reversible encephalopathy syndrome: incidence of atypical regions of involvement and imaging findings. AJR Am J Roentgenol 2007;189:904-12.

50 Eichler FS, Wang P, Wityk RJ, et al. Diffuse metabolic abnormalities in reversible posterior leukoencephalopathy syndrome. AJNR Am J Neuroradiol 2002;23:833-7.

51 Covarrubias DJ, Luetmer PH, Campeau NG. Posterior reversible encephalopathy syndrome: prognostic utility of quantitative diffusion-weighted MR images. AJNR Am J Neuroradiol 2002;23:1038-48.

52 Rahmlow MR, Kantarci O. Fulminant demyelinating diseases. Neurohospitalist 2013:3:81-91.

53 Caldemeyer KS, Smith RR, Harris TM, et al. MRI in acute disseminated encephalomyelitis. Neuroradiology 1994;36:216-20.

54 Campi A, Filippi M, Comi G, et al. Acute transverse myelopathy: spinal and cranial MR study with clinical follow-up. AJNR Am J Neuroradiol 1995;16:115-23.

55 Balasubramanya KS, Kovoor JM, Jayakumar PN, et al. Diffusion-weighted imaging and proton MR spectroscopy in the characterization of acute disseminated encephalomyelitis. Neuroradiology 2007;49:177-83.

56 Dalmau J, Gleichman AJ, Hughes EG, et al. Anti-NMDA-receptor encephalitis: case series and analysis of the effects of antibodies. Lancet Neurol 2008;7:1091-8.

57 Duffey P, Yee S, Reid IN, et al. Hashimoto's encephalopathy: postmortem findings after fatal status epilepticus. Neurology 2003;61:1124-6.

58 Gultekin SH, Rosenfeld MR, Voltz R, et al. Paraneoplastic limbic encephalitis: neurological symptoms, immunological findings and tumour association in 50 patients. Brain 2000;123(Pt 7):1481-94.

59 Lawn ND, Westmoreland BF, Kiely MJ, et al. Clinical, magnetic resonance imaging, and electroencephalographic findings in paraneoplastic limbic encephalitis. Mayo Clin Proc 2003; 78:1363-8.

60 Basu S, Alavi A. Role of FDG-PET in the clinical management of paraneoplastic neurological syndrome: detection of the underlying malignancy and the brain PET-MRI correlates. Mol Imaging Biol 2008;10:131-7.

61 Yoneda M. [Diagnosis and treatments of Hashimoto's encephalopathy]. Rinsho Shinkeigaku 2012;52:1240-2.

62 Mahad DJ, Staugaitis S, Ruggieri P, et al. Steroid-responsive encephalopathy associated with autoimmune thyroiditis and primary CNS demyelination. J Neurol Sci 2005;228:3-5.

$63 \mathrm{Su} \mathrm{TH}$, Jin EH, He W. Hashimoto encephalopathy: a case report with proton MR spectroscopic findings. Chin Med J (Engl) 2011;124:3420-2.

64 Zhao W, Li J, Wang J, et al. A case of Hashimoto encephalopathy: clinical manifestation, imaging, pathology, treatment, and prognosis. Neurologist 2011;17:141-3.

65 Hsu CS, Wu YC, Lu MK, et al. 99mTc ECD and 99mTc TRODAT-1 SPECT in Hashimoto encephalopathy. Clin Nucl Med 2012;37:e86-8.

66 Fuertes J, Garcia-Burillo A, Castell-Conesa J, et al. Functional neuroimaging in Hashimoto's encephalitis: a physiopathological imaging? Eur J Nucl Med Mol Imaging 2009;36:726.

67 Aurelius $E$, Johansson B, Skoldenberg $B$, et al. Encephalitis in immunocompetent patients due to herpes simplex virus type 1 or 2 as determined by type-specific polymerase chain reaction and antibody assays of cerebrospinal fluid. J Med Virol 1993:39:179-86.

68 Casrouge A, Zhang SY, Eidenschenk $C$, et al. Herpes simplex virus encephalitis in human UNC-93B deficiency. Science 2006;314:308-12.

69 Sili U, Kaya A, Mert A, et al. Herpes simplex virus encephalitis: clinical manifestations, diagnosis and outcome in 106 adult patients. J Clin Virol 2014;60:112-18.
70 Baringer JR. Herpes simplex infections of the nervous system. Neurol Clin 2008;26:657-74, viii.

71 Hasegawa Y, Morishita M, Ikeda T, et al. Early diagnosis of herpes simplex virus encephalitis by single photon emission computed tomography (SPECT) in patients with normal MRI. Rinsho Shinkeigaku 1996;36:475-80.

72 Riancho J, Delgado-Alvarado M, Sedano MJ, et al. Herpes simplex encephalitis: clinical presentation, neurological sequelae and new prognostic factors. Ten years of experience. Neurol Sci 2013;34:1879-81.

73 Taira N, Kamei S, Morita A, et al. Predictors of a prolonged clinical course in adult patients with herpes simplex virus encephalitis. Intern Med 2009;48:89-94.

74 Mekan SF, Wasay M, Khelaeni B, et al. Herpes simplex encephalitis: analysis of 68 cases from a tertiary care hospital in Karachi, Pakistan. J Pak Med Assoc 2005;55:146-8.

75 Susac JO, Hardman JM, Selhorst JB. Microangiopathy of the brain and retina. Neurology 1979;29:313-16.

76 Allmendinger AM, Spektor V, Destian S. CT and MR imaging of Susac syndrome in a young male presenting with acute disorientation. Clin Imaging 2010;34:138-42.

77 Gross M, Eliashar R. Update on Susac's syndrome. Curr Opin Neurol 2005:18:311-14.

78 White ML, Zhang Y, Smoker WR. Evolution of lesions in Susac syndrome at serial MR imaging with diffusion-weighted imaging and apparent diffusion coefficient values. AJNR Am J Neuroradiol 2004:25:706-13.

79 Dielman C, Laureys $G$, Meurs A, et al. Susac syndrome: a case report and PET imaging findings. Acta Neurol Belg 2009;109:226-30.

80 Puoti G, Bizzi A, Forloni $G$, et al. Sporadic human prion diseases: molecular insights and diagnosis. Lancet Neurol 2012;11:618-28.

81 Hayashi R, Hanyu N, Kuwabara T, et al. Serial computed tomographic and electroencephalographic studies in Creutzfeldt-Jakob disease. Acta Neurol Scand 1992;85:161-5

82 Schroter A, Zerr I, Henkel K, et al. Magnetic resonance imaging in the clinical diagnosis of Creutzfeldt-Jakob disease. Arch Neurol 2000;57:1751-7.

83 Shiga Y, Miyazawa K, Sato S, et al. Diffusion-weighted MRI abnormalities as an early diagnostic marker for Creutzfeldt-Jakob disease. Neurology 2004;63:443-9.

84 Vitali P, Maccagnano E, Caverzasi E, et al. Diffusion-weighted MRI hyperintensity patterns differentiate CJD from other rapid dementias. Neurology 2011;76:1711-19.

85 Collins SJ, Sanchez-Juan P, Masters CL, et al. Determinants of diagnostic investigation sensitivities across the clinical spectrum of sporadic Creutzfeldt-Jakob disease. Brain 2006;129(Pt 9):2278-87.

86 Collie DA, Summers DM, Sellar RJ, et al. Diagnosing variant Creutzfeldt-Jakob disease with the pulvinar sign: MR imaging findings in 86 neuropathologically confirmed cases. AJNR Am J Neuroradiol 2003;24:1560-9.

87 Yi SH, Park KC, Yoon SS, et al. Relationship between clinical course and Diffusion-weighted MRI findings in sporadic Creutzfeldt-Jakob disease. Neurol Sci 2008;29:251-5

88 Srivastava T, Kadam N. Bilateral putaminal hemorrhagic necrosis with rapid recovery of sensorium in a patient with methanol intoxication. J Postgrad Med 2013:59:243-4.

89 Ari S, Caca I, Kayabasi H. Bilateral complete optic atrophy and hemorrhagic infarction of the putamen caused by methanol intoxication. Ann Ophthalmol (Skokie) 2007;39:249-52.

90 Caparros-Lefebvre D, Policard J, Sengler C, et al. Bipallidal haemorrhage after ethylene glycol intoxication. Neuroradiology 2005;47:105-7. 